\title{
Wirksamkeit eines spezifisch für Zwangsstörungen entwickelten stationären Gruppentherapiekonzepts bei gleichzeitiger Verhaltenstherapie und pharmakologischer Behandlung
}

\section{The Efficacy of an Inpatient Group Therapy Program for Obsessive-Compulsive Disorder in Addition to Individual Cognitive Behavioral Therapy and Medication}
D. Althaus ${ }^{a}$
M. Zaudig ${ }^{b}$
W. Hauke
G. Röper ${ }^{\mathrm{C}}$
W. Butolloc

aPsychiatrische Klinik der Ludwig-Maximilians-Universität, München, bPsychosomatische Klinik Windach,

${ }^{\mathrm{c}}$ Institut für Klinische Psychologie, Ludwig-Maximilians-Universität München

\section{Schlüsselwörter \\ Zwangsstörung · Kognitive Verhaltenstherapie · Spezifisches Gruppentherapiekonzept}

\section{Zusammenfassung}

Zielsetzung: Ziel dieser Studie war die Untersuchung, inwieweit Patienten mit der Erstdiagnose Zwangsstörung (ICD 10, F 42) von einem speziellen Gruppentherapiekonzept profitieren, das zusätzlich zu verhaltenstherapeutischer Einzeltherapie und optionaler Pharmakotherapie zur Anwendung kommt.

Methoden: 30 Patienten wurden nach einem Zufallsverfahren der Experimental- ( $E G, \mathrm{n}=14$ ) oder der Kontrollgruppe (KG, $\mathrm{n}=16$ ) zugeordnet. Teilnehmer der EG erhielten neben einer kognitiv-behavioralen Einzeltherapie zusätzlich eine tägliche zwangsspezifische Gruppentherapie. Die KG erhielt neben kognitiv-behavioraler Einzeltherapie als Kontrollbedingung eine unspezifische Gruppentherapie. 7 Patienten erhielten zusätzlich eine Behandlung mit selektiven Serotonin-WiederaufnahmeHemmern (SSRIs; $E G=3 ; K G=4$ ). Vor Therapiebeginn und nach Therapieende wurden Y-BOCS, BDI und STAI-X1 erhoben. Darüber hinaus kamen Therapeuten-Ratings und Selbstbeurteilungen zum Einsatz.

Ergebnisse: In beiden Gruppen erzielten die Patienten hochgradige Besserungen der Zwangssymptomatik. Die Y-BOCS sank in der EG im Mittelwert von 23,64 auf 11,35 Punkte, in der KG von 23,81 auf 12,81 Punkte. Es fanden sich keine signifikanten Gruppenunterschiede $(\alpha=0,05)$ bei klinischen Maßen, Therapeuten-Ratings und Selbstbeurteilungen. In der EG waren signifikant mehr Responder als in der KG (Kriterium: Y-BOCS-Verbesserung $>50 \%, p=0,034)$. Zudem findet sich eine um 15 Tage geringere Behandlungsdauer.

Schlussfolgerungen: Eine Überlegenheit des Gruppenkonzepts konnte nicht bestätigt werden. Dennoch wurde damit ein zusätzlicher Therapiebaustein gefunden, der zu einer Ökonomisierung der Behandlung (kürzere Verweildauer) beiträgt und zu einem sehr großen Anteil hochgradig gebesserter Patienten führt.

\section{Key Words}

Obsessive Compulsive Disorder · Cognitive-behavioral treatment for OCD . Specific group therapy for OCD

\section{Summary}

Purpose: The goal of this treatment trial was to investigate the efficacy of an inpatient group therapy program specifically designed for patients suffering from Obsessive Compulsive Disorder (OCD, ICD 10, F 42).

Methods: In the experimental group (EG; $\mathrm{n}=14$ ) the specific group therapy was carried out in addition to individual cognitive-behavioral therapy, the control group (KG; $n=16$ ) received non-specific group therapy in mixed patient-groups in addition to individual therapy. 7 patients $(E G=3, K G=4)$ received also Selective Serotonin re-uptake inhibitors (SSRIs). Y-BOCS, BDI, and STAI-X1 were performed pre- and post-treatment. Furthermore therapists' ratings and self ratings were taken.

Results: At post-treatment both groups showed significant improvement of the symptomatology. For the EG the Y-BOCS mean was reduced from 23.64 to 11.35 , for the KG from 23.81 to 12.81. The group comparison showed no significant differences $(\alpha=0.05)$ in clinical measurements, therapists' and self ratings. However, there were significantly more responders (improvement $>50 \%$, Y-BOCS) in the EG and treatment duration was shorter by 15 days.

Conclusions: Clinical outcome in the EG was not superior. However, the specific group therapy for OCD contributes to an economic and effective treatment.

\begin{tabular}{ll}
\hline KARGER & @ 2000 S. Karger GmbH, Freiburg \\
$\begin{array}{l}\text { Fax +49 7614520714 } \\
\begin{array}{l}\text { E-mail Information@Karger.de } \\
\text { www.karger.com }\end{array}\end{array}$ & Accessible online at: \\
www.karger.com/journals/ver
\end{tabular}

\section{KARGER}

le online at:

www.karger.com
David Althaus

Psychiatrische Klinik der Ludwig-Maximilians-Universität

Nußbaumstraße 7

D-80336 München (Deutschland) 


\section{Einleitung}

Behaviorale Verfahren haben sich seit den frühen Arbeiten von Meyer [1966] in einer Vielzahl von Studien als effektive Behandlungsmethode bei Zwangsstörungen erwiesen [Röper und Rachman, 1980; Marks et al., 1975; Rachman und Hodgson, 1980; v. Balkom et al., 1994; Stanley und Turner, 1995]. Im Zentrum der Behandlung steht die Durchführung von Konfrontationsübungen (ERV: Exposition und Reaktionsverhinderung, eine ausführliche Darstellung des Verfahrens findet sich unter anderem bei Marks [1987], Hand [1992] und Reinecker [1994]). Der Mehrzahl der Patienten kann dabei langfristig und dauerhaft geholfen werden [Übersicht bei Abramowitz, 1997]. Stanley und Turner [1995] kommen in einer Literaturdurchsicht zum Ergebnis, dass selbst unter Berücksichtigung von Therapieverweigerern und -abbrechern $63 \%$ der Patienten positiv auf eine verhaltenstherapeutische Behandlung ansprechen (Symptomreduktion > 30\%, Y-BOCS). Trotz dieser erfreulichen Therapieergebnisse kann nicht übersehen werden, dass aufgrund mäßiger psychosozialer Versorgung durch verhaltenstherapeutisch geschulte Behandler [Reinecker, 1994, S.111] nur eine kleine Minderzahl der zwangsgestörten Patienten - epidemiologische Untersuchungen ermitteln eine 6-Monats-Prävalenz zwischen 1 und 2\% [Rasmussen, 1994] - angemessen unterstützt werden kann. Einer enormen Anzahl behandlungsbedürftiger Patienten steht nur eine sehr begrenzte Menge an Therapieplätzen gegenüber. Um so erstaunlicher ist die Tatsache, dass die klinische Forschung der Möglichkeit von kostensparenden gruppentherapeutischen Behandlungskonzepten bei Zwangsstörungen bislang kaum Beachtung geschenkt hat. Die wenigen dazu veröffentlichten Studien zeigen heterogene Befunde [Hand und Tichatzky, 1979; Espie, 1986; Krone et al., 1991; Enright, 1991; Fals-Stewart et al., 1993; Münchau et al., 1996] und lassen keine klaren Rückschlüsse zu, inwieweit die Gruppenbehandlung einen Beitrag bei der Therapie von Zwangsstörungen leisten kann. Zudem ist den meisten Arbeiten nicht zu entnehmen, ob Besserungseffekte speziell durch die Gruppenbehandlung erzielt wurden oder Folge von anderen Therapiemaßnahmen waren.

Die bislang einzige größere und kontrollierte Studie hinsichtlich der Wirksamkeit von Gruppenbehandlung bei Zwangsstörungen wurde von Fals-Stewart, Marks und Schaefer [1993] mit 93 ambulanten zwangsgestörten Patienten durchgeführt (3 Behandlungsbedingungen: Einzeltherapie; Gruppentherapie; progressive Muskelentspannung). Nach 12wöchiger Therapiedauer und im Follow-up (6 Monate) zeigten sich sowohl verhaltenstherapeutische Einzelwie auch Gruppenbehandlung erfolgreich (durchschnittliche Symptomreduktion etwa $40 \%$, Y-BOCS). Es fanden sich keine signifikanten Unterschiede zwischen beiden Bedingungen. Einschränkend muss festgehalten werden, dass die Patientenstichprobe dieser Studie einer starken Selektion unterworfen war. So wurden nur solche Patienten zugelas- sen, die weder eine komorbide Persönlichkeitsstörung noch eine zusätzliche Depression hatten.

Ziel dieser Studie ist es daher, herauszufinden, ob sich mit einer zusätzlichen eigens für Zwangsstörungen konzipierten Gruppenbehandlung, die neben individueller Verhaltenstherapie und optionaler Pharmakotherapie zum Einsatz kommt, ein zusätzlicher Effekt nachweisen lässt.

\section{Design und Hypothesen der Studie}

Patienten der Psychosomatischen Klinik Windach mit der Erstdiagnose Zwangsstörung nach ICD-10 [Internationale Klassifikation psychischer Störungen, Dilling et al., 1992] wurden nach einem Zufallsprinzip in zwei Gruppen aufgeteilt. Patienten der Experimentalgruppe (EG) erhalten neben einer verhaltenstherapeutischen Individualtherapie, die ein Konfrontationstraining beinhaltet, eine intensive eigens für Zwangsstörungen konzipierte Gruppenbehandlung. Patienten der Kontrollgruppe (KG) erhalten neben der Einzeltherapie zusätzlich eine störungsunspezifische Gruppenbehandlung, die spezielle für Zwangsstörungen relevante Fragen nicht berücksichtigt. Auf diese Art soll erreicht werden, Gruppenunterschiede zwischen EG und KG tatsächlich auf das zusätzliche Modul einer zwangsstörungsspezifischen Gruppentherapie zurückführen zu können. Die Störungsvariablen Depression, Angst und Zwangssymptomatik werden zu drei Messzeitpunkten (Aufnahme, Entlassung, Follow-up) erfasst; zudem werden Therapeuten-Ratings und Selbstbeurteilungen ausgewertet. Es wird erwartet, dass sich die EG nach der Behandlung und im Follow-up (Katamnesezeitraum 12-14 Monate) in allen Besserungsmaßen als überlegen erweist.

\section{Behandlungssetting und Gruppenkonzept}

In der Psychosomatischen Klinik Windach wird ein multimodaler verhaltenstherapeutischer Behandlungsansatz [Lazarus 1978] verfolgt, der sich am Selbstmanagement-Therapiekonzept von Kanfer Reinecker und Schmelzer [1996] orientiert [Zaudig et al., 1998]. Im Zentrum steht dabei eine zielorientierte Einzeltherapie, die je nach Indikation von anderen Therapiemodulen ergänzt werden kann (unter anderem Selbstsicherheitstraining, Entspannungstechniken, Gestaltungstherapie, Pharmakotherapie). Als Schlüsselfaktor für die Behandlung von Zwangsstörungen wird die intensive Durchführung von Konfrontationsübungen (ERV) angesehen, die sich meist in Form einer gezielten Reizüberflutung mit Reaktionsverhinderung («flooding», vgl. Marks [1987]) über 2 Wochen erstreckt. Neben einer Habituation wird dabei insbesondere ein modifizierter, kompetenter Umgang mit bislang vermiedenen Gefühlen intendiert [Hand, 1994; Hauke, 1998; Hauke und Niedermeier, 1998].

Patienten aus der EG nehmen neben der Einzeltherapie an einer spezifisch für Zwangsstörungen entwickelten Gruppentherapie teil. Dabei erhalten die Patienten (je Gruppe maxi- 
mal 8 Personen) pro Woche 5 gemeinsame Sitzungen, von denen eine in Eigenregie ohne therapeutische Begleitung durchgeführt wird, die anderen 4 Termine finden unter therapeutischer Leitung statt. Wesentliche Faktoren des Gruppenkonzepts sind:

- Intensiver Erfahrungsaustausch und gegenseitige Unterstützung der Patienten.

- Die Vermittlung eines für die Patienten verstehbaren Erklärungsmodells für Genese und Aufrechterhaltung der Störung (behavioral-kognitive und biologische Komponenten).

- Schulung der Selbstwahrnehmung (Identifikation eigener körperlicher, kognitiver und behavioraler Reaktionsmuster). Die Patienten führen beispielsweise von Anfang an Protokoll über kleine Konfrontationsübungen, um so selbst besser den Ablauf und die Funktionalität der eigenen Störung zu begreifen.

- Verbesserung der emotionalen Wahrnehmungsfähigkeit. In diesem Zusammenhang kommt den emotional aktivierenden Verfahren (unter anderem Rollenspiele, Gestaltdialoge und Familienskulpturen) übergeordnete Bedeutung zu. Vor dem theoretischen Hintergrund, dass ritualistisches Verhalten letztlich ein Vermeidungsverhalten von unangenehmen Gefühlen (Angst, Unsicherheit, Schuld, Aggressivität) darstellt [vgl. Rachman und Hodgson, 1980], wird hier versucht, dem Patienten neue Erfahrungen und Reaktionsmöglichkeiten bezüglich seiner Gefühle zu ermöglichen.

- Identifikation und Bearbeitung von Auslösesituationen (situative, emotionale, familiäre), die der Störung zugrunde liegen.

- Vorbereitung von Flooding-Maßnahmen. Für jeden Patienten werden individuell geeignete Übungen zusammengestellt.

- Erarbeitung von Strategien zum Umgang mit dem Zwang.

- Förderung sozialer Kompetenzen (unter anderem durch Rollenspiele). Bei zwangsgestörten Patienten finden sich häufig gravierende Defizite im kommunikativen Bereich (Ausdruck eigener Bedürfnisse und Gefühle, Selbstbehauptung, Umgang mit Ärger und Wut usw.).

- Förderung rückfallpräventiver Maßnahmen.

Patienten aus der Kontrollgruppe nahmen statt dessen 2mal pro Woche an einer störungsunspezifischen Gruppentherapie $(\mathrm{n}=8-10)$ teil. Die anderen Patienten in diesen Gruppen litten größtenteils unter Depressionen, Angststörungen, Anpassungsstörungen, Persönlichkeitsstörungen und Somatisierungsstörungen. Hier wurden Fragen zu Zwang nicht thematisiert. Ziel dieser Gruppen war die Vermittlung allgemeiner Problemlösestrategien und der Erwerb sozialer Kompetenzen.

\section{Methoden}

Yale-Brown-Obsessive-Compulsive-Scale (Y-BOCS) [Goodman et al., 1989; Beschreibung und deutsche Bearbeitung in Hand und Büttner-
Westphal, 1991]. Die Y-BOCS gilt in der englischsprachigen Forschung seit einigen Jahren als Standard-Rating-Verfahren der Wahl zur qualitativen Spezifizierung und Quantifizierung des Schweregrads von Zwangsstörungen.

Beck-Depressions-Inventar (BDI) [Beck et al., 1961; deutsche Version: Hautzinger, 1991]

State-Trait-Anxiety Inventory (STAI) [Spielberger et al., 1970; deutsche Fassung: Laux et al., 1980]. In der vorliegenden Studie wurde die Zustandsangst (20 Items) gemessen (STAI-X1; State-Angst).

\section{Basisdokumentation}

Die Basisdokumentation beinhaltet zahlreiche demografische Variablen (Familienstand, Schulbildung, Beruf usw.), Angaben zur Art der Störung (genaue Diagnose nach ICD-10, Erkrankungsdauer usw.), Informationen über frühere und jetzige Behandlungen (Differenzierung nach einzelnen Behandlungselementen) sowie über Medikation bei Aufnahme und Entlassung.

\section{Therapeuten-Rating}

Bei Entlassung fand eine Einschätzung der klinischen und symptomatischen Besserung ( 1 = wesentlich verbessert bis 7 = wesentlich verschlechtert) und der weiteren Prognose statt $(1=$ günstig; 2 = zweifelhaft; 3 = ungünstig).

\section{Selbst-Rating}

Ein Selbst-Rating wird in der Klinik Windach routinemäßig vom Patienten kurz vor der Entlassung ausgefüllt. Dabei schätzt der Patient unter anderem auf einer 5-Stufen-Skala den subjektiven Therapieerfolg ein ( 1 = sehr gut, 3 = befriedigend, bis $5=$ schlecht $)$.

\section{Medikation}

Ein Teil der Patienten erhielt optional neben der psychotherapeutischen Behandlung Psychopharmaka; der Einsatz erfolgte nach klinischen Gesichtspunkten. Die eingesetzten Psychopharmaka wurden folgenden Untergruppen zugeordnet: SSRIs, Trizyklische Antidepressiva, Tranquillizer, Neuroleptika.

\section{Statistische Methodik}

Die statistische Auswertung erfolgte mittels SPSS [Brosius und Brosius 1995]. Alle Variablen wurden auf Normalverteilung und Varianzhomogenität getestet. Wo Testvoraussetzungen nicht erfüllt waren, wurden nonparametrische Verfahren durchgeführt. Wenn für Mittelwertsvergleiche (Students t-Test) die Voraussetzungen fehlten, kam der Mann-Whitney U-Test zum Einsatz, der auf der Basis von Ordinalskalen rechnet. Bei den Korrelationen wurde statt des Person-Koeffizienten gegebenenfalls auf die Spearman Rangkorrelation zurückgegriffen. Als Signifikanzniveau wurde $\alpha=0,05$ gewählt.

\section{Durchführung}

Bei dieser Untersuchung handelt es sich um eine naturalistische Studie, die in der Psychosomatischen Klinik Windach durchgeführt wurde. In die Studie wurden Patienten mit der Diagnose einer primären Zwangsstörung (ICD 10; F. 42.) einbezogen, die zwischen August 1996 und Juni 1997 behandelt worden waren. Die Patienten wurden nach einem Zufallsprinzip dem Gruppenkonzept oder der Kontrollgruppe zugewiesen und auf den verschiedenen Stationen von verhaltenstherapeutisch erfahrenen Ärzten und Diplom-Psychologen behandelt. Die Therapie wurde von pflegerischen Fachkräften co-therapeutisch unterstützt. Die Therapeuten hatten ein hausinternes Ausbildungsprogramm absolviert und wurden regelmäßig supervidiert, so dass die Homogenität der Behandlungsdurchführung gewährleistet war.

Die Y-BOCS wurde von therapeutischer Seite zu Beginn (t1) und am Ende der Behandlung (t2) erhoben. Darüber hinaus wurden bei Aufnahme und Entlassung BDI und STAI-X1 erfasst. Der Entlassungsbogen wurde am Ende des Aufenthalts in der Klinik Windach vom Patienten 
Tab. 1. Veränderung der Mittelwerte der klinischen Maße von $\mathrm{t} 1$ zu 2 in $\mathrm{EG}$ und $\mathrm{KG}$

\begin{tabular}{|c|c|c|c|c|c|c|c|c|}
\hline & \multicolumn{4}{|l|}{ EG } & \multicolumn{4}{|l|}{ KG } \\
\hline & MW (t1) & MW (t2) & Diff. & $\mathrm{p}$ (t-Test) & MW (t1) & MW (t2) & Diff. & $\mathrm{p}$ (t-Test) \\
\hline BDI & 18,36 & 11,79 & 6,57 & $0,004 * *$ & 19,31 & 15,31 & 4,00 & $0,040 *$ \\
\hline STAI-X1 & 57,31 & 48,31 & 10,00 & $0,041^{*}$ & 58,40 & 49,67 & 8,73 & 0,073 \\
\hline Y-BOCS & 23,64 & 11,35 & 12,28 & $0,000 * * *$ & 23,81 & 12,81 & 11,00 & $0,000 * * *$ \\
\hline
\end{tabular}

$*=\mathrm{p}<0,05, * *=\mathrm{p} \leq 0,01, * * *=\mathrm{p} \leq 0,001$ (einseitig).
Tab. 2. Vergleich von EG und KG bezüglich der durchschnittlichen Symptomreduktion

\begin{tabular}{|c|c|c|c|c|c|c|c|}
\hline & \multicolumn{2}{|l|}{ EG } & \multicolumn{2}{|l|}{ KG } & \multirow[t]{2}{*}{ Diff. } & \multirow[t]{2}{*}{$\mathrm{t}$-Wert } & \multirow[t]{2}{*}{$\mathrm{p}$} \\
\hline & MW & $\mathrm{n}$ & MW & $\mathrm{n}$ & & & \\
\hline \multicolumn{8}{|c|}{ BDI-Reduktion } \\
\hline Punkte & 6,57 & 14 & 4,00 & 16 & 2,57 & 1,00 & 0,18 \\
\hline$\%$ & 34,57 & 14 & 24,24 & 16 & 10,33 & 0,63 & 0,27 \\
\hline \multicolumn{8}{|c|}{ STAI-X1-Reduktion } \\
\hline Punkte & 9,00 & 13 & 7,53 & 15 & 1,46 & 0,24 & 0,40 \\
\hline$\%$ & 14,31 & 13 & 14,19 & 15 & 0,12 & 0,01 & 0,50 \\
\hline \multicolumn{8}{|c|}{ Y-BOCS-Reduktion } \\
\hline Punkte & 12,28 & 14 & 11,00 & 16 & 1,28 & 0,61 & 0,28 \\
\hline$\%$ & 52,06 & 14 & 48,25 & 16 & 3,82 & 0,43 & 0,33 \\
\hline
\end{tabular}

ausgefüllt. Nach der Entlassung erfolgte in der Basisdokumentation die therapeutische Einschätzung der symptomatischen Besserung sowie der Gesamtbesserung. Die Follow-up-Erhebung wird derzeit ausgewertet.

\section{Beschreibung der Stichprobe}

Von Anfang August bis Anfang Dezember 1996 wurden in der Klinik Windach 43 Patienten mit der Erstdiagnose Zwangsstörung (ICD 10; F 42.) aufgenommen. 5 der 43 aufgenommenen Patienten haben die Behandlung nach kurzer Zeit wieder abgebrochen oder waren nicht bereit, wesentlichen Teilen des Behandlungskonzeptes zu folgen. Sie weigerten sich insbesondere, Konfrontationsübungen durchzuführen und konnten daher nicht in die Studie aufgenommen werden. Weitere 3 Patienten erhielten zwar eine umfassende Behandlung, wurden jedoch nicht ausreichend diagnostisch dokumentiert, um an der statistischen Auswertung der Studie teilnehmen zu können.

Als Einschlusskriterium («Cut-off-Schwelle») wurde für gleichzeitig vorliegende Handlungs- und Gedankenzwänge ein Gesamtscore von 16 und mehr Punkten auf der Yale-Brown-Obsessive-Compulsive-Scale (Y-BOCS) festgelegt [Hand und Büttner-Westphal, 1991]. Für ausschließliche Handlungs- oder Gedankenzwänge gilt als Eingangskriterium dagegen ein Wert von 10 Y-BOCS-Punkten. Von den verbleibenden 35 Patienten hatten 5 Personen Eingangswerte von weniger als 16 (bzw. 10) Y-BOCS-Punkten, so dass schließlich 30 Patienten in die Studie aufgenommen werden konnten.

Die ICD-10-Diagnosen wurden routinemäßig nach klinischen Gesichtspunkten gestellt. Die Untersucher hatten regelmäßiges Training in ICD10-Diagnostik. Bei den Teilnehmern der Studie handelt es sich um 12 Frauen und 18 Männer mit einem Durchschnittsalter von 35,9 Jahren (Standardabweichung SD = 12,4; Bereich $\mathrm{r}=18-66$ Jahre). 10 von ihnen $(33,3 \%)$ litten unter vorwiegenden Handlungszwängen (F 42,1, Y-BOCSMW: 20,9), 16 (53,4\%) unter gemischten Zwängen (Handlungs- und Gedankenzwänge, F 42,2, Y-BOCS-MW: 26,6) und 4 (13,3\%) unter vorwie- genden Gedankenzwängen (F 42,0, Y-BOCS-MW: 19,3). 10 Patienten $(33,3 \%)$ hatten eine Zweitdiagnose (v.a. Persönlichkeitsstörungen und Dysthymia). Die mittlere Störungsdauer betrug 13,0 Jahre ( $\mathrm{SD}=11,7$; $r=2-57$ Jahre); das durchschnittliche Alter bei Beginn der Störung lag bei 23,1 Jahren.

\section{Ergebnisse}

\section{Schweregrad der Störung zu t1 und demografische Variablen}

Die beiden Gruppen (EG versus KG) unterschieden sich zu Beginn der Behandlung in keinem klinischen Maß signifikant. BDI $(\mathrm{MW}=18,36$ versus 19,31), STAI-X1 (MW = 57,31 versus 58,40) und Y-BOCS (MW = 23,64 versus 23,81) waren in beiden Gruppen annähernd identisch ausgeprägt. Ferner unterschieden sich die beiden Gruppen nicht signifikant hinsichtlich Zweitdiagnosen sowie psychotherapeutischer und medikamentöser Vorbehandlung. Bezüglich Geschlecht, Bildungsgrad, beruflichem Status, Familien- und Partnersituation und Arbeitsfähigkeit gab es zwischen den Gruppen keine signifikanten Unterschiede. Bei der Variable «Alter» zeigte sich im t-Test allerdings eine deutliche Tendenz $(\mathrm{p}=0,11)$ : das Durchschnittsalter der Patienten aus der EG lag mit 39,7 um 7,1 Jahre höher als das der Patienten aus der KG. Noch deutlicher wirkt sich diese Unterschiedlichkeit bei der Störungsdauer aus. Hier erreicht der Unterschied mit 17,4 Jahren (EG) versus 9,1 Jahren $(\mathrm{KG})$ annähernd Signifikanz $(\mathrm{p}=0,054)$. 
Abb. 1. Prozentuale Symptomreduktion (Y-BOCS) der Einzelfälle in EG und KG.

Abb. 2. Vergleich der Responder-Quoten in EG $(\mathrm{n}=14)$ und $\mathrm{KG}(\mathrm{n}=16)$.

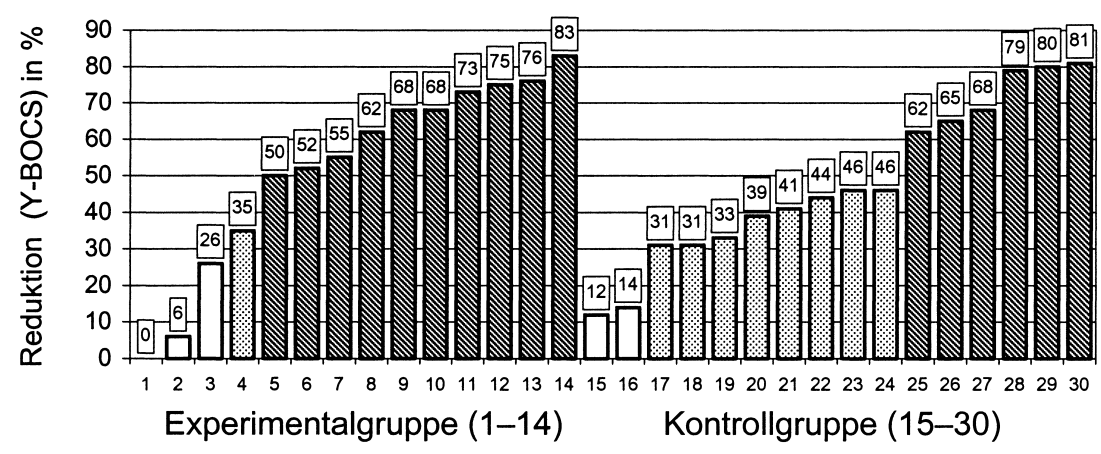

$\square$ Patienten mit einer Y-BOCS Reduktion von mind. 30\% Patienten mit einer Y-BOCS Reduktion von 30-49\% $\mathbb{N}$ Patienten mit einer Y-BOCS Reduktion von mind. 50\%

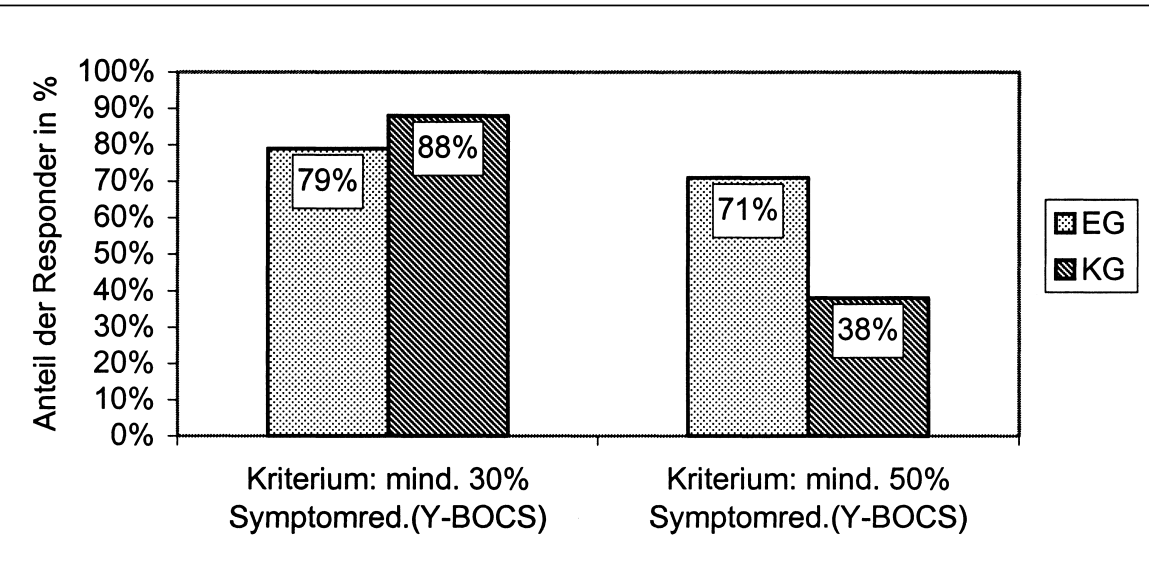

\section{Klinische Maße}

Sowohl in EG als auch in KG zeigen sich nach der Behandlung deutliche Besserungen hinsichtlich der klinischen Symptomatik. Insbesondere auf der Y-BOCS (Zwangssymptomatik) zeichnen sich für Patienten beider Gruppen deutliche Verbesserungen ab. Entgegen unserer Hypothese unterscheiden sich EG und KG in keinem klinischen Maß hinsichtlich der Symptomreduktion von t1 zu t2 (Tab. 1 und 2).

Die Reduktion der Zwangssymptomatik (Y-BOCS) beträgt in der EG 52\%, in der KG 48\%. Abbildung 1 zeigt die Reduktion der Y-BOCS für die einzelnen Patienten der jeweiligen Gruppen. Dabei fällt auf, dass sich EG und KG trotz der Ähnlichkeit der durchschnittlichen Symptomreduktion (52 versus $48 \%$ ) in ihren Profilen voneinander unterscheiden. Die KG weist eine sehr kontinuierliche Streuung der Besserungswerte auf, wobei $50 \%$ der Patienten im gemäßigten Bereich von $30-50 \%$ Symptomverbesserung zu finden sind. In der EG befindet sich nur ein Patient in diesem Bereich; für $71 \%$ der Patienten zeigt sich jedoch eine Symptomverbesserung von über
$50 \%$. Andererseits konnten in der EG zwei Patienten von der Behandlung auf Symptomebene gar nicht profitieren (0 bzw. $6 \%$ ). Diese deutliche Polarisierung in der EG (Patienten profitieren entweder gar nicht oder sehr gut) schlägt sich auch in den Responder-Quoten nieder (Abb. 2). Bei einer ResponderSchwelle von 30\% (mindestens 30\% Reduktion auf der Y-BOCS) zeigen sich entgegen der Untersuchungshypothese zwischen den Gruppen keine signifikanten Unterschiede ( $\mathrm{p}=$ 0,51; Mann-Whitney U-Test). Legt man jedoch das strengere Kriterium von $50 \%$ Symptomreduktion zugrunde, zeigt sich eine signifikante Überlegenheit für EG ( $p=0,034$; MannWhitney U-Test).

\section{Therapeuten-Ratings}

In den Therapeuten-Ratings finden sich entgegen unserer Hypothese für keine der Variablen bedeutsame Unterschiede zwischen den Gruppen (Tab. 3). 
Tab. 3. Vergleich von EG und $\mathrm{KG}$ bezüglich der Therapeuten-Ratings

\begin{tabular}{|c|c|c|c|c|c|}
\hline & \multicolumn{2}{|l|}{ EG } & \multicolumn{2}{|l|}{ KG } & \multirow[t]{2}{*}{ p (Mann-Whitney U-Test) } \\
\hline & MW & $\mathrm{n}$ & MW & $\mathrm{n}$ & \\
\hline Gesamtzustand & 2,143 & 14 & 2,400 & 15 & 0,126 \\
\hline Symptomatik & 2,071 & 14 & 2,267 & 15 & 0,174 \\
\hline Prognose & 1,357 & 14 & 1,467 & 15 & 0,213 \\
\hline Arbeitsfähigkeit & 1,167 & 14 & 1,142 & 15 & 0,869 \\
\hline Leistungsfähigkeit & 1,636 & 14 & 1,533 & 15 & 1 \\
\hline
\end{tabular}

\begin{tabular}{|c|c|c|c|c|}
\hline \multirow[t]{2}{*}{ Korrelationen (Person) } & \multicolumn{2}{|l|}{ Alter } & \multicolumn{2}{|c|}{ Störungsdauer } \\
\hline & $\mathrm{r}$ & $\mathrm{p}$ & $\mathrm{r}$ & $\mathrm{p}$ \\
\hline Y-BOCS Reduktion, \% $(\mathrm{n}=30)$ & $-0,05$ & 0,80 & 0,32 & 0,08 \\
\hline BDI Reduktion, \% $(\mathrm{n}=30)$ & 0,11 & 0,57 & 0,17 & 0,35 \\
\hline Therapeuten-Rating: Gesamtzustand $(\mathrm{n}=29)$ & $-0,13$ & 0,48 & $-0,36$ & $0,05^{*}$ \\
\hline Therapeuten-Rating: Symptomatik $(\mathrm{n}=29)$ & $-0,21$ & 0,26 & $-0,45$ & $0,02 *$ \\
\hline Subjektive Besserung $(\mathrm{n}=21)$ & $-0,39$ & 0,08 & $-0,42$ & 0,06 \\
\hline
\end{tabular}

$*=\mathrm{p} \leq 0,05$ (zweiseitig).

\section{Selbstbeurteilung des Therapieerfolgs durch die Patienten}

Leider wurde nur bei der Hälfte der Patienten aus der KG der sonst routinemäßig übliche Entlassungsbogen erfasst. Patienten, die den Entlassungsbogen nicht ausgefüllt hatten, unterschieden sich in den übrigen Besserungsmaßen nicht signifikant von den anderen Patienten der $\mathrm{KG}$, so dass die 8 Selbsteinschätzungen insgesamt als repräsentativ für die $\mathrm{KG}$ angesehen werden können. Bei der Beurteilung des Therapieerfolgs durch die Patienten finden sich entgegen unserer Hypothese keine signifikanten Unterschiede zwischen EG und KG ( $p=0,44$; Mann-Whitney U-Test). In der EG beurteilen 2 Patienten $(15,4 \%)$ den Therapieerfolg als sehr gut, 6 (46, $2 \%)$ als gut, $3(23,1 \%)$ als befriedigend und jeweils eine Person $(7,6 \%)$ als unbefriedigend oder schlecht. In der KG berichten jeweils 2 (25\%) Patienten über sehr guten, guten, befriedigenden und unbefriedigenden Erfolg.

\section{Der Einfluss der Variablen «Alter» und «Störungsdauer»}

Wie oben berichtet, unterschieden sich die beiden Gruppen deutlich (wenn auch nicht signifikant) hinsichtlich Alter und Störungsdauer. Da diese beiden Variablen in der Literatur mitunter als prognostisch ungünstig bezeichnet werden [vgl. Reinecker, 1994], wurden in dieser Studie ihre Korrelationen zu verschiedenen Besserungsmaßen errechnet, um so sicherstellen zu können, dass durch die Ungleichverteilung keine systematische Verzerrung der Ergebnisse stattgefunden hat. Dabei zeigte sich für die Gesamtstichprobe ( $\mathrm{n}=$
30), dass Alter und Störungsdauer mit einigen Besserungswerten mittelstark korrelierten und teilweise Signifikanz erreichten (Tab. 4). Entgegen der Annahme ging eine längere Störungsdauer dabei mit einem besseren Therapie-Outcome einher. Bei Betrachtung der Einzelgruppen zeigt sich, dass sich diese Korrelationen für EG und $\mathrm{KG}$ erheblich unterscheiden. Für die EG korreliert die Variable «Alter» mit keinem der Besserungswerte. Der Zusammenhang von Störungsdauer und Therapieerfolg ist dagegen für 3 der 5 verglichenen Besserungswerte signifikant. Subjektive Besserung $(\mathrm{r}=-0,72 ; \mathrm{p}=0,01)$, Therapeuten-Rating der symptomatischen Verbesserung $(\mathrm{r}=0,61 ; \mathrm{p}=0,02)$ und prozentuale Y-BOCS-Reduktion $(r=0,61 ; p=0,02)$ zeigen dabei mittlere bis hohe Korrelationskoeffizienten. Für die KG korreliert die Variable «Alter» signifikant mit subjektiver Besserung $(\mathrm{r}=-0,73 ; \mathrm{p}=0,039)$ und in negativer Richtung mit prozentualer Y-BOCS-Reduktion $(r=-0,55, p=0,029)$. Die Variable «Störungsdauer» zeigt in der KG keinen Zusammenhang zu einem Besserungswert (Tab. 5).

\section{Korrelationen zwischen ausgewählten Besserungsmaßen}

Zur Abschätzung der Konsistenz des Behandlungserfolgs wurden die Interkorrelationen der wichtigsten Besserungsmaße ermittelt. Für die EG korrelierten die verschiedenen Maße in einem hohen bis mittleren Bereich $(r=0,51-0,75)$ und zeigten Signifikanz. Für die KG konnten dagegen nur für die Korrelation von Y-BOCS-Reduktion und TherapeutenRating ( $r=0,51)$ Signifikanz errechnet werden (Tab. 6). 
Tab. 5. Korrelationen von Alter und Störungsdauer mit verschiedenen Besserungsmaßen (EG und $\mathrm{KG}$ )

\begin{tabular}{|c|c|c|c|c|c|c|c|c|}
\hline & \multicolumn{4}{|l|}{ EG } & \multicolumn{4}{|l|}{ KG } \\
\hline & \multicolumn{2}{|l|}{ Alter } & \multicolumn{2}{|c|}{ Störungsdauer } & \multicolumn{2}{|l|}{ Alter } & \multicolumn{2}{|c|}{ Störungsdauer } \\
\hline & $\mathrm{r}$ & $\mathrm{p}$ & $\mathrm{r}$ & $\mathrm{p}$ & $\mathrm{r}$ & $\mathrm{p}$ & $\mathrm{r}$ & $\mathrm{p}$ \\
\hline Y-BOCS Reduktion, \% & 0,09 & 0,77 & 0,61 & 0,02 & $-0,55$ & $0,03^{*}$ & $-0,05$ & 0,83 \\
\hline BDI Reduktion, \% & $-0,15$ & 0,61 & $-0,26$ & 0,37 & $-0,02$ & 0,61 & 0,11 & 0,69 \\
\hline $\begin{array}{c}\text { Therapeuten-Rating: } \\
\text { Gesamtzustand }\end{array}$ & $-0,19$ & 0,50 & 0,51 & 0,06 & 0,22 & 0,42 & $-0,02$ & 0,96 \\
\hline $\begin{array}{l}\text { Therapeuten-Rating: } \\
\text { Symptomatik }\end{array}$ & $-0,20$ & 0,50 & $-0,61$ & $0,02 *$ & 0,01 & 0,98 & 0,21 & 0,44 \\
\hline Subjektive Besserung & $-0,24$ & 0,43 & $-0,72$ & $0,01 * *$ & $-0,73$ & $0,04 *$ & $-0,31$ & 0,44 \\
\hline
\end{tabular}

$*=\mathrm{p} \leq 0,05, * *=\mathrm{p} \leq 0,01$ (zweiseitig).
Tab. 6. Korrelation von Y-BOCS- Reduktion, TherapeutenRating und Selbstbeurteilung des Therapieerfolgs in $\mathrm{EG}$ und $\mathrm{KG}$

\begin{tabular}{|c|c|c|c|c|c|c|c|}
\hline & \multirow[t]{2}{*}{ Spearman Rangkorrelation } & \multicolumn{3}{|c|}{ Therapeuten-Rating (Gesamtzustand) } & \multicolumn{3}{|c|}{ Y-BOCS-Reduktion } \\
\hline & & $\mathrm{r}$ & $\mathrm{p}$ & $\mathrm{n}$ & $\mathrm{r}$ & $\mathrm{p}$ & $\mathrm{n}$ \\
\hline \multirow[t]{2}{*}{ EG } & Y-BOCS-Reduktion, \% & $-0,75$ & $0,001 * * *$ & 14 & & & \\
\hline & subjektive Besserung & 0,73 & $0,003 * *$ & 13 & 0,51 & $0,038^{*}$ & 13 \\
\hline \multirow[t]{2}{*}{ KG } & Y-BOCS-Reduktion, \% & $-0,53$ & $0,020 * *$ & 15 & & & \\
\hline & subjektive Besserung & 0,00 & 0,500 & 8 & 0,34 & 0,204 & 8 \\
\hline
\end{tabular}

$*=\mathrm{p} \leq 0,05, * *=\mathrm{p} \leq 0,01, * * *=\mathrm{p} \leq 0,001$ (einseitig).

\section{Die Auswertung behandlungsbezogener Daten}

Patienten der EG wurden hypothesenkonform signifikant kürzer behandelt als Patienten der KG (78,1 Tage versus 94,3 Tage; $\mathrm{p}=0,049$, t-Test). Gleichzeitig war aufgrund der häufigeren Gruppensitzungen die Gesamtanzahl therapeutischer Anwendungen je Patient in der EG höher als in der KG (80,21 versus 64,9 Behandlungseinheiten, $p=0,026$; t-Test, eine Behandlungseinheit umfasste entweder 50 min Einzeltherapie oder 60-90 min Gruppentherapie).

\section{Medikamentöse Therapie}

Insgesamt hatten 13 Patienten bei Aufnahme eine psychopharmakologische Medikation (davon 9 SSRIs, 3 Trizyklika, 4 Tranquillizer, 2 Neuroleptika). Bei Entlassung hatten insgesamt 9 Patienten eine Medikation, 7 davon ein SSRI. EG und KG unterschieden sich weder in Häufigkeit, Dauer noch Art der Medikation voneinander.

\section{Diskussion}

Für die Mehrzahl der Variablen konnten unsere Hypothesen von den zusätzlichen Effekten eines spezifischen Gruppentherapiekonzepts für Zwangsstörungen keine Bestätigung finden.
Im Mittelwertsvergleich zeigte sich in keinem Besserungsmaß ein signifikanter Unterschied zwischen den beiden Gruppen. Beide Gruppen zeigten ein hohes Maß an Besserung. Die durchschnittliche Punktreduktion auf der Y-BOCS differierte zwischen den Gruppen nur um 1,28 Punkte zugunsten der EG. Selbst wenn sich bei höheren Fallzahlen diese Unterschiedlichkeit als stabil erweisen sollte, und Signifikanz erreicht würde, ist zu bezweifeln, dass diese Differenz klinische Relevanz besitzt.

Vergleicht man jedoch die einzelnen Fälle der beiden Gruppen bezüglich der prozentualen Symptomreduktion, so werden für die Y-BOCS unterschiedliche Profile sichtbar. Offenbar polarisiert die Behandlung in der EG die Patienten stärker: sie neigen dazu, sich im Therapieverlauf als «Top oder Flop» zu entwickeln; in der KG sind dagegen vor allem mittlere Verlaufsformen zu beobachten. Diese unterschiedlichen Profile schlagen sich wie oben dargestellt (Abb. 1) auch in den Responder-Quoten nieder. So erreichen in der EG signifikant mehr Patienten ein Behandlungsergebnis, das man als hochgradig gebessert bezeichnen kann (Symptomreduktion $>50 \%$ ).

Die durchschnittliche Behandlung in der EG dauerte signifikant kürzer als in der KG (72 versus 87 Tage). Eine um 15 Tage kürzere Behandlungsdauer ist gerade aus Wirtschaftlichkeitserwägungen sicherlich ein wichtiges Argument für die Behandlung innerhalb des Gruppenkonzepts. Dieses Ergebnis könnte damit zusammenhängen, dass aufgrund der 
zeitsparenden Gruppenbehandlung in kürzerer Zeit ein größeres Angebot an qualifizierten Therapieeinheiten möglich war.

Leider unterschieden sich die beiden Gruppen tendenziell in Bezug auf Alter und Störungsdauer. Versuche, den Einfluss dieser beiden Variablen zu bestimmen, brachten erstaunliche Ergebnisse zum Vorschein. So zeigte sich für die Gesamtstichprobe $(\mathrm{n}=30)$ der Befund, dass Alter und Störungsdauer eher Prädiktoren für einen guten Therapie-Outcome darstellten. Andererseits waren die Zusammenhänge in EG und $\mathrm{KG}$ höchst unterschiedlich. Während in der EG Patienten mit einer längeren Störungsdauer überproportional profitierten und das Alter keine Rolle spielte, zeigte sich in der Kontrollgruppe die Störungsdauer als irrelevant, das Alter der Patienten schien dagegen negativ mit dem Behandlungsergebnis korreliert. Möglicherweise zeigen die unterschiedlichen Behandlungskonzepte bei verschiedenen Patiententypen differentielle Effekte. Antworten darauf sind aufgrund der niedrigen Fallzahlen und der Heterogenität der Stichprobe gegenwärtig spekulativ. Es bleibt zunächst abzuwarten, ob sich diese Unterschiede im Follow-up und bei Replikationen bestätigen. Auch die Korrelationen der wichtigsten Besserungsmaße zeigen für die beiden Gruppen divergierende Befunde. Die für das Gruppenkonzept errechneten hohen bis mittleren Korrelationen dokumentieren einen konsistenten Therapieerfolg. Die Selbsteinschätzung der Patienten, die Beurteilung durch die Therapeuten sowie die gemessene Reduktion der Hauptsymptomatik hängen eng miteinander zusammen. Für die KG dagegen findet sich diese Konsistenz nicht. Besonders das Fehlen jeglichen Zusammenhangs zwischen Selbst- und Fremdbeurteilung des Therapieerfolgs fällt ins Auge. Hier haben Therapeuten und Patienten offenbar unterschiedliche Kriterien zugrunde gelegt. Um so mehr erstaunt es, dass die Patienten der KG bei den Absolutwerten der Symptomreduktion genauso gut abgeschnitten haben wie die der EG. Offenbar haben hier die Techniken zur Symptomreduktion (v.a. Konfrontationsübungen in Form des Floodings) ebenso gut gegriffen wie beim Gruppenkonzept. Allerdings bleibt abzuwarten, ob sich diese guten Ergebnisse über lange Sicht als stabil erweisen können. Die Follow-up Messung wird derzeit ausgewertet

\section{Zusammenfassung}

Die Hypothese der Überlegenheit des Gruppenkonzepts konnte in wichtigen Teilen nicht bestätigt werden. Dennoch bleibt festzuhalten, dass mit der spezifischen Gruppentherapie für Zwangsstörungen ein zusätzlicher Therapiebaustein gefunden wurde, der zu einer Ökonomisierung der Behandlung (z.B. kürzere Verweildauer) beiträgt und zu einem sehr hohen Anteil hochgradig gebesserter Patienten führt.

\section{Literatur}

Abramowitz JS: Effectiveness of psychological and pharmacological treatments for OCD: A quantitative review. J Consult Clin Psychol 1997;65:44-52.

v. Balkom AJLM, Oppen P, Vermeulen AWA, Nauta MMC, Vorst HCM, v. Dyck R: A meta-analysis on the Treatment of OCD. A comparison of anti-depressants, behavior and cognitive therapy. Clinical Psychol Review 1994;14:359-381.

Beck AT: Psychometric properties of the Beck Depression Inventory. 25 years of evaluation. Clin Psychol Rev 1988:8:77-100.

Brosius G, Brosius F: SPSS Base System and Professional Statistics. International Thomson Publishing. Kempten, Kösel, 1995.

Dilling H, Mombour M, Schmidt MH (Hrsg): World Health Organisation: ICD-10 Kapitel V (F). Internationale Klassifikation psychischer Störungen. Göttingen, Huber, 1992.

Enright SJ: Group treatment for OCD. An evaluation. Behav Psychother 1991;19:183-192.

Espie CA: The group treatment of obsessive- compulsive ritualisers: Behavioral management of identified patterns of relapse. Behav Psychother 1986;14:21-33.

Fals-Stewart W: A comparison of behavioral group therapy and individual behavior therapy in treating OCD. J Mental Nervous Dis 1993;181:189-193.

Goodman W, Rasmussen SA, Price LH, Mazure C, Heninger GR, Charney DS: The Yale-Brown Obsessive Compulsive Scale (Y-BOCS): Development, use and reliability. Arch Gen Psychiatry 1989;46:10061016.
Hand I: Verhaltenstherapie der Zwangsstörungen: Therapieverfahren und Ergebnisse; in Hand I, Goodman WK, Evers U (Hrsg): Zwangsstörungen. Neue Forschungsergebnisse. Berlin, Springer, 1992.

Hand I: Expositions-Reaktions-Management. Praxis klinischen Verhaltensmedizin und Rehabilitation 1994; 26:64-68

Hand I, Büttner-Westphal H: Die Yale-Brown Obsessive Compulsive Scale (Y-BOCS): Ein halbstrukturiertes Interview zur Beurteilung des Schweregrads von Denk- und Handlungszwängen. Verhaltenstherapie 1991;1:223-225.

Hand I, Tichatzky M: Behavioral group therapy for Obsessions and Compulsions: First results of a pilot study; in Sjöden PO (ed): Trends in Behavior Therapy. New York, Academic Press, 1979.

Hauke W: Verhaltenstherapie bei Zwangsstörungen; in Zaudig M, Hegerl U, Hauke W (Hrsg): Zwangsstörungen. Stuttgart, Schatthauer, 1998

Hauke W, Niedermeier N: Verhaltensanalyse und funktionales Bedingungsmodell bei Zwangsstörungen in Zaudig M, Hegerl U, Hauke W (Hrsg): Zwangsstörungen, Stuttgart, Schatthauer, 1998

Hautzinger M: Das Beck Depressions Inventar (BDI ) in der Klinik. Nervenarzt 1991;62:689-696.

Kanfer FH, Reinecker H, Schmelzer D: Selbstmanagement-Therapie (2. Ausg). Berlin, Springer, 1996.

Krone KP, Himle JA, Nesse R: A standardized behavioral program for OCD: preliminary outcomes. Behaviour Research and Therapy 1991;29:253-257.

Laux L: Das State-Trait-Angstinventar. Theoretische Grundlagen und Handanweisung. Weinheim, Beltz, 1980.
Lazarus A: Multimodale Verhaltenstherapie. Frankfurt/M., Fachbuchhandlung für Psychologie, 1978. Marks IM: Fears, Phobias and Rituals. Panic, Anxiety and their Disorders. New York, Oxford University Press, 1987

Meyer V: Modifikation of expectations in cases with obsessional rituals. Behaviour Research and Therapy 1966;4:273-280.

Münchau N, Hand I, Schaibl R, Lotz C: Aufbau von Selbsthilfegruppen für Zwangskranke unter verhaltenstherapeutischer Expertenanleitung: Empirische Ergebnisse. Verhaltenstherapie 1996;6:143-160.

Rachman S, Hodgson RJ: Obsessions and Compulsions. New York, Prentice-Hall, 1980.

Rasmussen SA, Eisen JL: The epidemiology and differential diagnosis of obsessive compulsive disorder. J Clin Psychiatry 1994;55(suppl):5-10.

Reinecker H: Zwänge. Diagnose, Theorien und Behandlung. Göttingen, Huber, 1994.

Röper G, Rachman S: Obsessional-compulsive checking: Experimental replication and development. Behav Res Ther 1976;14(1):25-32.

Spielberger CD: Manual for the State-Trait Anxiety Inventory. Palo Alto CA, Consulting Psychologis Press, 1970.

Stanley AM, Turner SM: Current status of pharmacological and behavioral treatment of OCD. Behavior Therapy 1995;26:163-186.

Zaudig M, Hauke W, Hegerl U: Die Zwangsstörung. Diagnostik und Therapie. Stuttgart, Schattauer, 1998. 